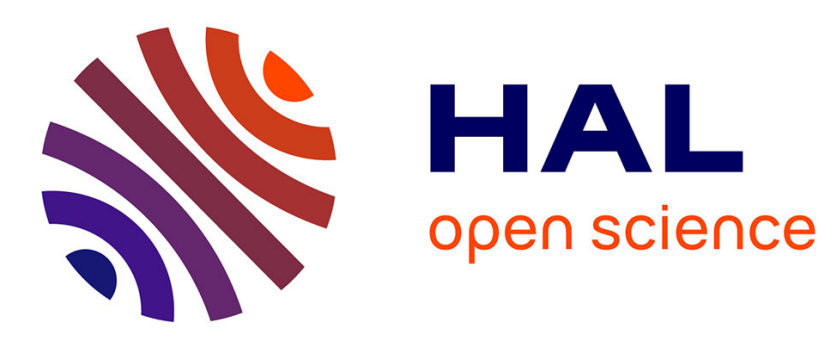

\title{
Resultants and Discriminants for Bivariate Tensor-product Polynomials
}

Angelos Mantzaflaris, Elias Tsigaridas

\section{To cite this version:}

Angelos Mantzaflaris, Elias Tsigaridas. Resultants and Discriminants for Bivariate Tensor-product Polynomials. ISSAC 2017 - International Symposium on Symbolic and Algebraic Computation, Mohab Safey El Din, Jul 2017, Kaiserslautern, Germany. pp.8, 10.1145/3087604.3087646 . hal-01525560

\author{
HAL Id: hal-01525560 \\ https://hal.inria.fr/hal-01525560
}

Submitted on 21 May 2017

HAL is a multi-disciplinary open access archive for the deposit and dissemination of scientific research documents, whether they are published or not. The documents may come from teaching and research institutions in France or abroad, or from public or private research centers.
L'archive ouverte pluridisciplinaire HAL, est destinée au dépôt et à la diffusion de documents scientifiques de niveau recherche, publiés ou non, émanant des établissements d'enseignement et de recherche français ou étrangers, des laboratoires publics ou privés. 


\section{Resultants and Discriminants for Bivariate Tensor-product Polynomials}

\author{
Angelos Mantzaflaris \\ Johann Radon Institute for Computational and \\ Applied Mathematics (RICAM), \\ Austrian Academy of Sciences \\ Altenberger Str. 69, 4040, Linz, Austria \\ angelos.mantzaflaris@oeaw.ac.at
}

\author{
Elias Tsigaridas \\ Sorbonne Universités, UPMC Univ Paris 06, \\ CNRS, INRIA, \\ Laboratoire d'Informatique de Paris 6 (LIP6), \\ Équipe POLSYS, \\ 4 place Jussieu, 75252 Paris Cedex 05, France \\ elias.tsigaridas@inria.fr
}

\begin{abstract}
Optimal resultant formulas have been systematically constructed mostly for unmixed polynomial systems, that is, systems of polynomials which all have the same support. However, such a condition is restrictive, since mixed systems of equations arise frequently in practical problems. We present a square, Koszul-type matrix expressing the resultant of arbitrary (mixed) bivariate tensor-product systems. The formula generalizes the classical Sylvester matrix of two univariate polynomials, since it expresses a map of degree one, that is, the entries of the matrix are simply coefficients of the input polynomials. Interestingly, the matrix expresses a primaldual multiplication map, that is, the tensor product of a univariate multiplication map with a map expressing derivation in a dual space. Moreover, for tensor-product systems with more than two (affine) variables, we prove an impossibility result: no universal degree-one formulas are possible, unless the system is unmixed. We present applications of the new construction in the computation of discriminants and mixed discriminants as well as in solving systems of bivariate polynomials with tensor-product structure.
\end{abstract}

\section{CCS Concepts}

-Computing methodologies $\rightarrow$ Algebraic algorithms;

\section{Keywords}

Koszul resultant matrix, tensor-product, polynomial system solving, separation bounds, mixed discriminant

\section{INTRODUCTION}

One of the central problems in elimination theory is the construction of determinantal formulas for the resultant. There is also special emphasis on exploiting the sparsity, or in other words the support, of the input equations.

We refer the reader to [32] as a basic reference, to [13] for a nice introduction to the theory of resultants, to [10] for its application in Computer-aided Geometric Design (CAGD),

Permission to make digital or hard copies of all or part of this work for personal or classroom use is granted without fee provided that copies are not made or distributed for profit or commercial advantage and that copies bear this notice and the full citation on the first page. Copyrights for components of this work owned by others than the author(s) must be honored. Abstracting with credit is permitted. To copy otherwise, or republish, to post on servers or to redistribute to lists, requires prior specific permission and/or a fee. Request permissions from permissions@acm.org.

ISSAC '17, July 25-28, 2017, Kaiserslautern, Germany

(C) 2017 Copyright held by the owner/author(s). Publication rights licensed to ACM ISBN 978-1-4503-5064-8/17/07 . \$ \$15.00

DOI: http://dx.doi.org/10.1145/3087604.3087646 and to [27] for various matrix constructions. The optimal construction that one can hope for is a degree-one formula; that is a matrix whose non-zero entries are coefficients of the input polynomials (modulo multiplication with \pm 1 ), and whose determinant is equal to the resultant. The Sylvestertype formulas fall in this category. Unfortunately, such formulas do not always exist for given Newton polytopes. There are also Bézout-type formulas where the entries of the matrix are coefficients of the Bezoutian polynomial, which have high degree with respect to the input polynomials coefficients. We call the matrices that have both types of blocks hybrid. For general algorithms for computing resultant matrices, let us mention [19] that proposes a rational resultant formula (quotient of two determinants) for the mixed homogeneous case, and [18] that generalizes Macaulay's construction [36] to the sparse case, as well as the tools in [12, 28, 29].

Our focus is on resultants and discriminants for polynomial systems in two variables. A polynomial system is called unmixed if all of its members have the same Newton polytope, and mixed otherwise. Exact resultant formulas are mostly known for certain classes of unmixed systems, and very little is known for the general mixed case. We are interested in optimal (or exact) degree-one formulas for the mixed resultant. Degree-one formulas refer to matrices where the entries are coefficients of the input polynomials (possibly with a sign change). This kind of expressions are very convenient for both the analysis and the implementation of resultant methods, since the matrix entries are simple to compute and have known bitsize. Common degree-one formulas are the Sylvester-type formulas; in this work we present a different one, which expresses a second order Koszul map.

Khetan presented explicit exact formulas for an arbitrary unmixed sparse bivariate polynomial system [34]. His determinantal formula is a hybrid Sylvester and Bézout type. Also in the unmixed case there are necessary and sufficient conditions for the Dixon resultant formulation to produce the resultant $[15,16]$. In the same context, Elkadi and Galligo proposed in [23] to use a variable substitution and two iterated resultants to compute the resultant polynomial.

Regarding Sylvester-type formulas, in [50] matrices expressing optimally the resultant of unmixed bivariate polynomials with corner-cut support are found. Moreover, Sylvester formulas for more general unmixed bivariate systems were depicted in [35]. The proof of the main theorem makes use of tools from algebraic geometry, including sheaf cohomology on toric varieties and Weyman's resultant complex. 
There are also methods for constructing resultant matrices for bivariate polynomial systems that combine Sylvester type blocks with toric Jacobian blocks in the case where the Newton polytopes of the polynomials are scaled copies of a single polygon, see [20] and references therein. The determinant of these matrices is a multiple of the sparse resultant, that is, the formula may not be optimal.

Resultants are closely related to discriminants. Discriminants have many applications, ranging from singularity theory of partial differential equations to the computation of the Voronoï diagram of curved objects [14, 25, 31]. Especially for the bivariate case we refer to [22], and references therein, that relates the mixed discriminant with the sparse resultant and the toric Jacobian.

Tensor-product systems fall in the general category of multihomogenenous systems [46]. In [42] and [39] one finds the first expressions of the resultant of such systems as the determinant of a matrix.

For unmixed multigraded systems Sturmfels and Zelevinski provided in [45] optimal Sylvester-type formulas. These formulas arise as certain choices of a Weyman complex of modules [32, 47, 48]. Many, if not all, classical resultant matrices are instances of such complexes [49], including the projective resultant [19]. In [21, 26] there is a systematic exploration of possible determinantal complexes, and also a software package that produces formulas for unmixed (and even scaled) resultants. Interestingly, there is a plethora of hybrid resultant matrices that consist of Bézout-type and Sylvester-type blocks [21, 26], see also [17].

The main contributions of this work are as follows. We present a determinantal degree-one formula for the resultant of arbitrary (mixed) bivariate tensor-product systems (Theorem 3.2). The formula applies without any restrictions on the bidegree of the polynomials, it expresses a Koszul map and has degree one with respect to the coefficients of the system. Moreover, we prove that the univariate and the bivariate case are the only cases among tensor-product polynomials which admit an unconditional formula of degree one (Lemma 3.3). We provide a constructive method to compute the new matrix, by identifying the (dual) multiplication maps, therefore making our formula explicit (Theorem 4.1). We call the matrix Koszul resultant matrix. We consider two applications of our matrix. Firstly, we use the Koszul resultant matrix to compute the discriminant of a bivariate tensor-product polynomial, and the mixed discriminant of arbitrary bivariate tensor-product systems (Lemma 5.1). Secondly, we use the $u$-resultant approach for computing roots of bivariate tensor-product systems. In particular, using the new resultant matrix at hand, we are able to augment any square system by a bilinear polynomial, and therefore efficiently obtain a rational univariate representation of the roots, as well as the coordinate multiplication maps of the bivariate ideal via a Schur complement.

The rest of the paper is organized are follows. In the next section we present some preliminary results that we need for our construction. In Section 3 we present the mixed resultant complex for the bivariate case and we derive the determinantal Koszul formula. Moreover, we show that universal degree-one formulas arise for at most two variables. In Section 4 we provide the algorithmic construction of the Koszul resultant matrix, by identifying the cohomology groups which appear in the complex. Finally, in Section 5 we apply the new matrix to the problem of computing dis- criminants and mixed discriminants, as well as to bivariate system solving. Throughout the paper, several toy examples accompany the main results.

\section{PRELIMINARIES}

Let $S_{1}\left(b_{1}\right), S_{2}\left(b_{2}\right)$ be the linear space of univariate polynomials of degree $b_{1}$ and $b_{2}$, in $\mathbb{K}[x]$ and $\mathbb{K}[y]$, respectively, where $\mathbb{K}$ is an algebraically closed field. The tensor-product space $S_{1}\left(b_{1}\right) \otimes S_{2}\left(b_{2}\right) \subset \mathbb{K}[x, y]$ consists of all polynomials of bidegree $\left(b_{1}, b_{2}\right)$. Throughout the paper we consider three arbitrary bivariate tensor-product polynomials

$$
f_{0}, f_{1}, f_{2} \in \mathbb{K}[x, y], \text { with } f_{i} \in S_{1}\left(d_{i 1}\right) \otimes S_{2}\left(d_{i 2}\right)
$$

and we denote by $\boldsymbol{d}_{i}=\left(d_{i 1}, d_{i 2}\right)$ the bidegree of $f_{i}, i=0,1,2$. In matrix notation, each polynomial is written as

$$
f_{i}(x, y)=\left(1, x, x^{2}, \ldots, x^{d_{i 1}}\right) F_{i}\left(1, y, y^{2}, \ldots, y^{d_{i 2}}\right)^{T},
$$

where $F_{i} \in \mathbb{Z}^{\left(d_{i 1}+1\right) \times\left(d_{i 2}+1\right)}, i=0,1,2$ are the coefficient matrices.

Assuming that the square system $f_{i}=f_{j}=0$ is zero-dimensional, the number of roots in $\mathbb{P}^{1} \times \mathbb{P}^{1}$ is equal to the Bézout bound

$$
D=D\left(f_{i}, f_{j}\right):=d_{i 1} d_{j 2}+d_{j 1} d_{i 2},
$$

where $D$ appears as the coefficient of the multilinear term of a certain polynomial [40, Theorem 2]. This number is the mixed volume of the system, and, in case of infinitely many roots, it is equal to the degree of the variety [24].

ExAmple 2.1. Consider two tensor-product polynomials of degrees $\boldsymbol{d}_{1}=(1,2)$ and $\boldsymbol{d}_{2}=(2,1)$, i.e.

$$
\begin{aligned}
& f_{1}=b_{1,2} x y^{2}+b_{0,2} y^{2}+b_{1,1} x y_{1}+b_{0,1} y+b_{1,0} x+b_{0,0} \\
& f_{2}=c_{2,1} x^{2} y+c_{1,1} x y+c_{2,0} x^{2}+c_{0,1} y_{1}+c_{1,0} x+c_{0,0} .
\end{aligned}
$$

The number of roots of the system in $\mathbb{P}^{1} \times \mathbb{P}^{1}$ is $D\left(f_{1}, f_{2}\right)=5$.

We fix the bidegrees in (1); we consider the vector space

$$
\mathcal{V}:=\bigoplus_{i=0}^{2} S_{1}\left(d_{i 1}\right) \otimes S_{2}\left(d_{i 2}\right) \cong \prod_{i=0}^{2} \mathbb{P}^{d_{i 1}} \times \mathbb{P}^{d_{i 2}}
$$

and we assume that it yields a very ample vector bundle of rank 3 on the irreducible projective variety $X=\mathbb{P}^{1} \times \mathbb{P}^{1}[32]$. We consider the incidence variety

$\mathcal{W}=\left\{\left(F_{0}, F_{1}, F_{2}, x, y\right) \in \mathcal{V} \times \mathbb{P}^{1} \times \mathbb{P}^{1}: f_{i}(x, y)=0, i=0,1,2\right\}$ and the projection $\pi: \mathcal{W} \rightarrow \mathcal{V}$. The image

$$
\pi(\mathcal{W})=\left\{\left(F_{0}, F_{1}, F_{2}\right): \exists(x, y), f_{i}(x, y)=0\right\}
$$

is called the resultant variety, and is an irreducible hypersurface in $\mathcal{V}$. The equation $\mathcal{R}\left(f_{0}, f_{1}, f_{2}\right)=0$ of this hypersurface characterizes the systems of the form (1) with common roots in $X$. This polynomial of $\mathbb{Z}[\mathcal{V}]$ is the resultant of $f_{0}, f_{1}, f_{2}$, it is homogeneous of degree $D\left(f_{i}, f_{j}\right)$ with respect to (the coefficients of) $f_{k},\{i, j, k\}=\{0,1,2\}$, and therefore it has total degree

$$
\operatorname{deg} \mathcal{R}\left(f_{0}, f_{1}, f_{2}\right)=\sum_{0 \leq i<j \leq 2} d_{i 1} d_{j 2}+d_{j 1} d_{i 2} .
$$

This resultant is an instance of the sparse resultant [32], where the toric variety is the product of two projective lines. Our aim is to obtain a square matrix, the entries of which are coefficients of the input polynomials (up to a sign change), that has determinant equal to the resultant. 


$$
\begin{aligned}
& \nu: \quad 3 \quad 2 \quad 1 \quad 0
\end{aligned}
$$

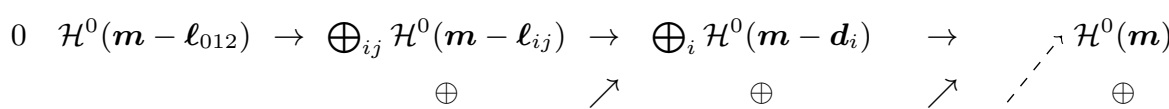

$$
\begin{aligned}
& 1 \\
& \mathcal{H}^{1}\left(\boldsymbol{m}-\boldsymbol{\ell}_{012}\right) \quad \rightarrow \bigoplus_{i j} \mathcal{H}^{1}\left(\boldsymbol{m}-\boldsymbol{\ell}_{i j}\right) \\
& \oplus
\end{aligned}
$$

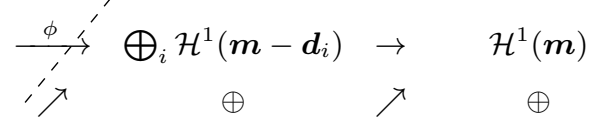

$$
\begin{aligned}
& \mathcal{H}^{2}\left(\boldsymbol{m}-\boldsymbol{\ell}_{012}\right) \quad \rightarrow \quad \bigoplus_{i j} \mathcal{H}^{2}\left(\boldsymbol{m}-\boldsymbol{\ell}_{i j}\right) \rightarrow \bigoplus_{i} \mathcal{H}^{2}\left(\boldsymbol{m}-\boldsymbol{d}_{i}\right) \rightarrow \mathcal{H}^{2}(\boldsymbol{m})
\end{aligned}
$$

Figure 1: The mixed Weyman complex of bivariate tensor-product polynomials. Whenever $i$ or $i j$ appear in a sum, they take values over all subsets of $\{0,1,2\}$ of 1 and 2 elements, respectively. The term $K_{\nu, q}$ in (4) is shown in row $q$ and column $\nu$ in this diagram. The resultant formula of Theorem 3.2 is the map $\phi: K_{1,1} \rightarrow K_{0,1}$, and is also marked in the diagram. For the sake of brevity we define $\boldsymbol{\ell}_{012}=\boldsymbol{d}_{0}+\boldsymbol{d}_{1}+\boldsymbol{d}_{2}$ and $\boldsymbol{\ell}_{i j}=\boldsymbol{d}_{i}+\boldsymbol{d}_{j}$. Determinantal formulas arise whenever $K_{2, q}=K_{-1, q}=0$, for all $q \in\{0,1,2\}$. The map $K_{\nu, q} \rightarrow K_{\nu-1, q^{\prime}}$ has degree one if and only if $q=q^{\prime}$, and is always zero if $q<q^{\prime}$, this is why no arrows point downwards in the diagram. The arrow between $K_{1,2}$ and $K_{0,0}$ is drawn with a dashed line for the sake of clarity.

\section{MIXED DETERMINANTAL COMPLEX}

In this section we elaborate on a mixed determinantal Weyman complex, which will help us arrive at the Koszul resultant matrix. The resultant polynomial can be computed as the determinant of the Weyman complex [47], see also [9], which arises by applying the, so called, geometric technique of [48] to the incidence variety of $f_{0}, f_{1}, f_{2}$ and generalizes the Cayley-Koszul complex [32]. Note that we cannot use the classical Koszul complex to derive resultant matrices for tensor-product (or, more generally, bigraded) polynomial systems (cf. [44]).

\subsection{The parameterized complex}

For any $\boldsymbol{m}=\left(m_{1}, m_{2}\right) \in \mathbb{Z}^{2}$ and $\left(f_{0}, f_{1}, f_{2}\right) \in \mathcal{V}$, Weyman's construction is a complex $K_{\bullet}=K_{\bullet}\left(m_{1}, m_{2}\right)$ of finite dimensional vector spaces:

$$
0 \rightarrow K_{3} \rightarrow K_{2} \rightarrow K_{1} \stackrel{\phi}{\rightarrow} K_{0} \rightarrow K_{-1} \rightarrow K_{-2} \rightarrow 0 .
$$

The terms are defined as $K_{\nu}=\oplus_{q=0}^{2} K_{\nu, q}$ with

$$
K_{\nu, q}=\bigoplus_{|I|=\nu+q} \mathcal{H}^{q}\left(\boldsymbol{m}-\sum_{i \in I} \boldsymbol{d}_{i}\right) \quad, \quad \nu=-2, \ldots, 3
$$

where $I$ runs over all subsets of $\{0,1,2\}$ of cardinality $\nu+q$. In Figure 1 the complex is shown (the indices $\nu, q$ refer to columns and rows, respectively). We use the notation $\boldsymbol{\ell}_{i j}$ and $\ell_{012}$ to denote sums of degrees of the $f_{i}$ 's.

Since we are working in the product of two projective lines, $X=\mathbb{P}^{1} \times \mathbb{P}^{1}$, the cohomology groups take the form

$$
\mathcal{H}^{q}(\boldsymbol{b})=H^{a_{1}}\left(b_{1}\right) \otimes H^{a_{2}}\left(b_{2}\right),
$$

with $a_{1}+a_{2}=q, \nu=-2, \ldots, 3$, and $H^{a}(b), b \in \mathbb{Z}$ denotes the $a$-th cohomology group of $\mathbb{P}^{1}$ with coefficients in the sheaf $\mathscr{O}(b)$ [33].

These terms depend solely on $m_{1}, m_{2}$. The maps between the terms (e.g. $\phi$ ) depend polynomialy on the coefficients of $f_{0}, f_{1}, f_{2}$. This construction appeared in [47] and a detailed presentation is given in [48].

The complex appears in Figure 1 in its full generality. However, several of the terms $K_{\nu, q}$ can be zero, but not all. We observe that for any integer vector $\boldsymbol{m}, K_{1} \neq 0$ and $K_{0} \neq 0$. Indeed, due to Bott's formula [3] the dimension of a cohomology group as a $\mathbb{K}$-vector space is

$$
\operatorname{dim} H^{a}(b)=\left((-1)^{a}(b+1)\right)_{+}, \quad a=0,1,
$$

where for an integer $q \in \mathbb{Z},(q)_{+}:=(q+|q|) / 2$.

Whenever this dimension is zero, it is understood $H^{a}(b)=$ 0 . This is the case for negative integers for global sections $(a=0)$, and for integers $b \geq-1$ for first order cohomologies.

The crucial property of the complex (3) is that its determinant is equal to (a power of) $R\left(f_{0}, \ldots, f_{2}\right)$. In general, this is a rational expression of determinants of maximal minors of differentials [32]. When the complex has only two non-zero terms (for specific integers $m_{1}, m_{2}$ ), then we obtain the resultant as the determinant of a single square matrix expressing the map $\phi$ at the non-zero part of the complex. We call such complexes and the induced square matrix expressions determinantal. The determinantal complexes are of the form $0 \rightarrow K_{1} \rightarrow K_{0} \rightarrow 0$, that is, $\nu \in\{0,1\}$.

The linear map $\phi$ is an epimorphism if and only if the complex is exact or, equivalently, the polynomials do not have a common root in $\mathbb{P}^{1} \times \mathbb{P}^{1}$. The possible values of $\left(m_{1}, m_{2}\right)$ which lead to determinantal complexes is a finite set $[21,26]$.

EXAMPLE 3.1. The resultant of three bilinear forms, that is, $\boldsymbol{d}_{0}=\boldsymbol{d}_{1}=\boldsymbol{d}_{2}=(1,1)$ corresponds to the determinantal complex $K_{\bullet}(2,1)$, i.e. $\boldsymbol{m}=(2,1)$. Using (12), the complex (3) becomes $K_{1,1} \stackrel{\phi}{\rightarrow} K_{0,0}$, and implies the existence of a map $\phi: S(1,0)^{\oplus 3} \rightarrow S(2,1)$ expressing the resultant. This resultant matrix is depicted in [21, Section 7.1].

Many classically known resultant formulas can be obtained as the determinant of different instances of $\phi$ in (3), for particular integers $\left(m_{1}, m_{2}\right) \in \mathbb{Z}^{2}$. Moreover, the existence of a determinantal complex implies a determinantal formula for the resultant.

A Sylvester map is a linear function $S: \mathcal{P} \rightarrow \mathcal{Q}$ and has the form

$$
\left(g_{0}, g_{1}, g_{2}\right) \mapsto g_{0} f_{0}+g_{1} f_{1}+g_{2} f_{2} .
$$

When $\operatorname{dim} \mathcal{P}=\operatorname{dim} \mathcal{Q}$ this map is a generically surjective linear operator which can be expressed as the matrix (in compact form)

$$
\left[\begin{array}{lll}
f_{0} & f_{1} & f_{2}
\end{array}\right]
$$


The determinant of this matrix is equal to $\mathcal{R}\left(f_{0}, f_{1}, f_{2}\right)$.

A Koszul map (see for instance [41]) $K: \mathcal{P} \rightarrow \mathcal{Q}$ of second order is a map of the form

$$
\left(g_{0}, g_{1}, g_{2}\right) \mapsto\left(-g_{0} f_{1}-g_{1} f_{2}, g_{0} f_{0}-g_{2} f_{2}, g_{1} f_{0}+g_{2} f_{1}\right)
$$

Similarly, in the case of a linear operator we shall recover the resultant of $f_{0}, f_{1}, f_{2}$ as the determinant of the matrix

$$
\left[\begin{array}{rrr}
-f_{1} & -f_{2} & 0 \\
f_{0} & 0 & -f_{2} \\
0 & f_{0} & f_{1}
\end{array}\right]
$$

In the next section we provide the construction of a general Koszul operator for bivariate tensor-product systems.

\subsection{The determinantal Koszul formula}

In this section we identify a determinantal complex with a linear differential $\phi$ which has degree one with respect to the coefficients of the input polynomials, and whose determinant is the resultant of the system. The matrix expresses a Koszul linear operator, thus the name Koszul formula.

We can obtain a non-hybrid determinantal formula if and only if the non-zero terms in (3) (also Figure 1) are

$$
0 \rightarrow K_{1, q} \stackrel{\phi}{\rightarrow} K_{0, q^{\prime}} \rightarrow 0
$$

for some $q, q^{\prime} \in\{0,1,2\}$ (cf. [49]). General such formulas for unmixed multilinear systems are identified in [21, 26, 45, 49]. In general, the degree of $\phi: K_{\nu, q} \rightarrow K_{\nu-1, q^{\prime}}$ with respect to $f_{0}, f_{1}, f_{2}$ is equal to $\left(q-q^{\prime}+1\right)+$ therefore degree one linear maps arise from $q=q^{\prime}$. The following theorem presents the degree vector and formula of a degree one $\left(q=q^{\prime}=1\right)$ determinantal complex for the systems in question.

Theorem 3.2. Consider the degree vector

$$
\boldsymbol{m}=\left(d_{01}+d_{11}+d_{21}-1,-1\right) .
$$

The non-zero part of the complex $K_{\bullet}(\boldsymbol{m})$ is the operator $\phi: K_{1,1} \rightarrow K_{0,1}$, that is,

$$
\phi: \bigoplus_{0 \leq i<j \leq 2} \mathcal{H}^{1}\left(\boldsymbol{m}-\boldsymbol{\ell}_{i j}\right) \rightarrow \bigoplus_{0 \leq i \leq 2} \mathcal{H}^{1}\left(\boldsymbol{m}-\boldsymbol{d}_{i}\right) .
$$

Proof. First we show that $\mathcal{H}^{2}(\boldsymbol{m})=\mathcal{H}^{0}(\boldsymbol{m})=0$ (cf. Figure 1). Indeed we note that, by $(5), H^{0}(b)$ vanishes for any $b \leq-1$, therefore the row $q=0$ vanishes if $m_{k} \leq-1$, for some $k$. Similarly, $H^{1}(b)$ vanishes for any $b \geq-1$, so the row $q=2$ vanishes if there exists $k$ s.t. $m_{k} \geq d_{0 k}+d_{1 k}+d_{2 k}-1$.

Therefore the complex is confined to the row $q=1$. We can repeat these arguments to see that the extremal terms of the row $q=1$ also vanish, that is, $\mathcal{H}^{1}\left(\boldsymbol{m}-\boldsymbol{\ell}_{012}\right)=$ $\mathcal{H}^{1}\left(-1,-d_{02}-d_{12}-d_{22}-1\right)=0$ and $\mathcal{H}^{1}(\boldsymbol{m})=0$. We are left with the map (9), which is actually an operator, as we can verify using Bott's formula (5).

We remark that another choice of degree vector is possible, that is, $\boldsymbol{m}=\left(-1, d_{02}+d_{12}+d_{22}-1\right)$, which leads to the transposed operator of (9) (essentially the same formula).

In the univariate case the resultant variety is determinantal; it is given by the classical Sylvester matrix of the form (6). We have just shown that, in the bivariate tensorproduct case, there exists a formula, which does not have the structure of (6). A natural question is whether any of these two (or any other) degree-one formulas are possible, unconditionally, for multivariate tensor-product systems.
LEMMA 3.3. For $n>2$, and for tensor-product polynomials $f_{0}, \ldots, f_{n}$ with $n$ variables there is no universal degree one determinantal formula for $\mathcal{R}\left(f_{0}, \ldots, f_{n}\right)$. That is, the only such formulas, without any assumption on the degrees $\boldsymbol{d}_{i}$ are the classical Sylvester map for $n=1$ and the Koszul map (9) for $n=2$.

Proof. For proving that the only universal degree one formulas formulas arise for $n \leq 2$, it suffices to depict a general family of polynomials for which no such formula is possible. To this end, consider a degree vector $\boldsymbol{v} \in \mathbb{Z}_{\geq 0}$ and let the degrees be $\boldsymbol{d}_{i}=s_{i} \boldsymbol{v}, 0 \leq i \leq n$, for some positive integers $s_{0}, s_{1}, \ldots, s_{n}$ (scaled Newton polytopes). Indeed, in [26, Theorem 4.11] it is proven that the only degree one ${ }^{1}$ formulas (up to transposition) for this class of systems arise for $n \leq 2$. These are the classical Sylvester map (for $n=1$ ) and the Koszul map of (9) (for $n=2$ ).

Nevertheless the determinantal Koszul formula does appear in certain cases for $n>2$. For example, for trivariate tensor-product polynomials of degrees $\boldsymbol{d}_{0}=\boldsymbol{d}_{1}=\boldsymbol{d}_{2}=$ $(1,1,2)$ and $\boldsymbol{d}_{3}=(1,1,4)$ the formula $(9)$ is determinantal, for $\boldsymbol{m}=(2,3,-1)$. However, if we change $\boldsymbol{d}_{3}$ to $(2,2,4)$ the system is scaled, and for instance the degree vector $\boldsymbol{m}=(2,4,-1)$ yields the determinantal complex

$$
\begin{aligned}
0 \rightarrow & \mathcal{H}^{1}(0,2,-5)^{\oplus 3} \oplus \mathcal{H}^{2}(-2,0,-9)^{\oplus 3} \\
& \rightarrow \mathcal{H}^{1}(1,3,-3)^{\oplus 3} \otimes \mathcal{H}^{1}(0,2,-5) \rightarrow 0
\end{aligned}
$$

which does contain a Koszul part, but also additional degree two differentials appear.

Finally, for the sake of completeness we briefly recall the (mixed) homogeneous case. Consider homogeneous bivariate polynomials of degrees $d_{0}, d_{1}, d_{2}$, in non-decreasing order. In [19, Lemma 5.3] it is shown that a determinantal formula is possible if and only if

$$
1+d_{0}+d_{1}-d_{2} \geq 0 \text {. }
$$

Moreover, [26, Theorem 4.11] applies, since this system is trivially in the class of systems with scaled Newton polytopes, and it implies that arbitrary mixed homogeneous systems do not admit any degree-one formulas, except if $n=1$.

If all the polynomials have the same bidegree then another set of $n$ ! Sylvester formulas exist, for any $n$. These were discovered in [45] and independently in [35] for the bivariate case. Let $\boldsymbol{d}_{0}=\boldsymbol{d}_{1}=\boldsymbol{d}_{2}=\left(a_{1}, a_{2}\right)$, so that $D\left(f_{i}, f_{j}\right)=$ $2 a_{1} a_{2}$; the Newton polytopes $\left(a_{1}, 2 a_{2}\right)$ and $\left(2 a_{1}, a_{2}\right)$ both have volume $2 a_{1} a_{2}$ and multiplication by $f_{i}$ results in polytopes $\left(2 a_{1}, 3 a_{2}\right)$ and $\left(3 a_{1}, 2 a_{2}\right)$ (respectively) with volume $6 a_{1} a_{2}$. In our terminology, the complex $K_{\bullet}\left(2 a_{1}, 3 a_{2}\right)$ is determinantal and of Sylvester-type. Lemma 3.3 implies that these degree vectors are not universal, that is, the assumptions of [35, Theorem 1] cannot be satisfied by arbitrary degree vectors $\boldsymbol{d}_{i}$. Moreover, our Koszul formula does not satisfy these assumptions; however, it provides an additional formula for the resultant of unmixed systems.

EXAMPLE 3.4. Consider three polynomials with common bidegree $\boldsymbol{d}_{0}=\boldsymbol{d}_{1}=\boldsymbol{d}_{2}=(2,3)$. There exist two determinantal Sylvester formulas, for $\boldsymbol{m}=(5,5)$ and $\boldsymbol{m}=(3,8)$ as well as the Koszul determinantal formula $\boldsymbol{m}=(5,-1)$. These three formulas all have (matrix) dimension $24 \times 24$, which is the same as the degree of the resultant as a polynomial in $\mathbb{Z}[\mathcal{V}]$.

${ }^{1}$ In [26] degree one formulas are denoted as "pure Sylvester". 


\section{KOSZUL-TYPE MATRIX ASSEMBLY}

In this section we provide the algorithmic construction of the Koszul resultant matrix.

\subsection{Primal-dual multiplication maps}

First, we define dual polynomial spaces. Let $S_{i}^{*}(d)$ be the dual space of $S_{i}(d)$, that is, the space of linear functionals

$$
\lambda: S_{i}(d) \rightarrow \mathbb{K}
$$

We focus on the $y$ variable $(i=2)$ for the rest of the presentation. This space is isomorphic to (evaluations of) polynomials in formal derivatives, formally $S_{2}^{*}(d) \cong \mathbb{R}\left[\partial_{y}\right]_{d}$, see $[37,38]$ and references therein for a detailed presentation. We use this identification to obtain a basis for $S_{2}^{*}(d)$. For a univariate polynomial $g \in \mathbb{K}[y]$ and for $j \in \mathbb{Z}_{\geq 0}$ we define the functionals (reciprocal monomials)

$$
\psi^{-j} \in \mathbb{R}\left[\partial_{y}\right] \quad \text { with } \quad \psi^{-j}(g):=\frac{1}{j !} \frac{\mathrm{d}^{j} g}{\mathrm{~d} y^{j}}(0),
$$

where in particular $\psi^{0}(g):=1_{*}(g)=g(0)$ is evaluation at 0 . This way we obtain a basis $S_{2}^{*}(d)=\left\langle 1_{*}, \psi^{-1}, \ldots, \psi^{1-d}\right\rangle$. The latter is a $\mathbb{K}[y]$-module, equipped with the operation

$$
y^{b} \cdot \psi^{-j}:= \begin{cases}\psi^{(b-j)} & , \text { if } b \leq j \\ 0 & , \text { otherwise. }\end{cases}
$$

Here $\psi^{-j}$ acts as the inverse of $y^{j}$ in $\mathbb{K}\left[\partial_{y}\right]$, and this representation of $S_{2}^{*}(d)$ is sometimes referred to as inverse system.

The building blocks of the Koszul operator are (tensor products of):

- multiplication maps

$$
M_{h_{1}}: S_{1}\left(a_{1}\right) \rightarrow S_{1}\left(a_{1}+b_{1}\right)
$$

with $g \mapsto h_{1} g$ expressing the multiplication by a polynomial $h_{1} \in S_{1}\left(b_{1}\right)$,

- adjoint multiplication maps

$$
M_{h_{2}}^{*}: S_{2}^{*}\left(a_{2}+b_{2}\right) \rightarrow S_{2}^{*}\left(a_{2}\right)
$$

with $\lambda \mapsto h_{2} \cdot \lambda$, expressing the action of a polynomial $h_{2} \in S_{2}\left(b_{2}\right)$, as defined in (10).

We now recall the tensor product of multiplication and adjoint multiplication. With the preceeding definitions, there exists a unique linear operator

$$
\delta_{h_{1}, h_{2}}: S_{1}\left(a_{1}\right) \otimes S_{2}^{*}\left(a_{2}+b_{2}\right) \rightarrow S_{1}\left(a_{1}+b_{1}\right) \otimes S_{2}^{*}\left(a_{2}\right)
$$

satisfying

$$
\delta_{h_{1}, h_{2}}\left(x^{i} \otimes \psi^{-j}\right)=M_{h_{1}}\left(x^{i}\right) \otimes M_{h_{2}}^{*}\left(\psi^{-j}\right) .
$$

This map inherits the properties of its components, most importantly injectivity or surjectivity. In what follows we will omit the " $\otimes$ " and ". " between monomials and reciprocal monomials for the sake of brevity. With this definition of the tensor-product operator, we obtain

$$
\left(x^{a} y^{b}\right)\left(x^{i} \psi^{-j}\right)= \begin{cases}x^{a+i} \psi^{(b-j)} & , \text { if } b \leq j \\ 0 & , \text { otherwise }\end{cases}
$$

The above action extends by linearity to any element of $S_{1}\left(a_{1}\right) \otimes S_{2}^{*}\left(a_{2}+b_{2}\right)$. Moreover, map $\delta_{x^{a}, x^{b}}$ expresses the multiplication by the monomial $x^{a} y^{b}$ in that tensor-product space; by linearity we can consider any $h \in S_{1}\left(b_{1}\right) \otimes S_{2}\left(b_{2}\right)$ and define $\delta_{h}$ using (11). We refer to maps of the form of $\delta_{h}$ as primal-dual multiplication maps, see also [32, Ch. 13].

\subsection{The Koszul resultant matrix}

We are now in position to construct the final matrix. The cohomology groups in (4) are identified as

$$
H^{a}(b) \cong \begin{cases}S_{i}(b) & , a=0 \text { and } b>-1 \\ S_{i}^{*}(-b-2) & , a=1 \text { and } b<-1 \\ 0 & , a \neq 0,1 \text { or } b=-1 .\end{cases}
$$

This identification will allow us to deduce monomial bases for $H^{a}(b)$ and to express the map $\phi$ between the modules of (9) as a matrix. Indeed, we define

$$
V_{i j}:=\mathcal{H}^{1}\left(\boldsymbol{m}-\boldsymbol{\ell}_{i j}\right) \cong S_{1}\left(d_{k 1}-1\right) \otimes S_{2}^{*}\left(d_{i 2}+d_{j 2}-1\right)
$$

with $i<j$ and $k$ such that $\{i, j, k\}=\{0,1,2\}$ and

$$
W_{k}:=\mathcal{H}^{1}\left(\boldsymbol{m}-\boldsymbol{d}_{k}\right) \cong S_{1}\left(d_{i 1}+d_{j 1}-1\right) \otimes S_{2}^{*}\left(d_{k 2}-1\right),
$$

where $\boldsymbol{m}$ as in (8). Consider the primal-dual multiplication maps (see paragraph 4.1)

$\delta_{i j}^{r}: V_{i j} \rightarrow W_{r}$, for all $i, j \in\{0,1,2\}, i<j$ and $r \in\{i, j\}$, expressing multiplication by $f_{s}$ where $\{s\}=\{i, j\} \backslash\{r\}$. We will use the same notation for $\delta_{i j}^{r}$ and its (monomial basis) matrix representation. We have the following

THEOREM 4.1. Let $f_{0}, f_{1}, f_{2}$ be bivariate tensor-product polynomials as in (1). The map

$$
\phi: V_{01} \oplus V_{02} \oplus V_{12} \rightarrow W_{0} \oplus W_{1} \oplus W_{2}
$$

given as a second order Koszul map (7), is, up to a sign change, equal to the resultant

$$
\mathcal{R}\left(f_{0}, f_{1}, f_{2}\right)= \pm \operatorname{det} \phi= \pm \operatorname{det}\left[\begin{array}{ccc}
-\delta_{01}^{0} & -\delta_{02}^{0} & 0 \\
\delta_{01}^{1} & 0 & -\delta_{12}^{1} \\
0 & \delta_{02}^{2} & \delta_{12}^{2}
\end{array}\right] .
$$

Proof. From the above discussion and the identification (12) it is clear that the map in Theorem 3.2 defines the operator (13). The corresponding matrix is square, generically non-singular, and equals the determinant of the complex $K_{\bullet}(\boldsymbol{m})$, where $\boldsymbol{m} \in \mathbb{Z}^{2}$ as in (8), therefore it is equal to $\mathcal{R}\left(f_{0}, f_{1}, f_{2}\right)$, up to a sign change.

EXAMPLE 4.2. We will compute the Koszul resultant matrix for the system of Example 2.1, augmented by a bilinear polynomial

$$
f_{0}=a_{1,1} x y+a_{0,1} y+a_{1,0} x+a_{0,0} .
$$

We have the bidegrees $\boldsymbol{d}_{0}=(1,1), \boldsymbol{d}_{1}=(1,2)$ and $\boldsymbol{d}_{2}=$ $(2,1)$. The set $f_{0}, f_{1}, f_{2}$ is an overdetermined system of equations. The resultant has total degree

$$
\operatorname{deg} \mathcal{R}\left(f_{0}, f_{1}, f_{2}\right)=D\left(f_{0}, f_{1}\right)+D\left(f_{0}, f_{2}\right)+D\left(f_{1}, f_{2}\right)=11
$$

so this will be the dimension of the Koszul-type matrix. We compute $\boldsymbol{m}=(3,-1)$ using (8) and (9) becomes

$$
\begin{aligned}
0 \rightarrow \mathcal{H}^{1}(1,-4) \oplus \mathcal{H}^{1}(0,-3) \oplus \mathcal{H}^{1}(0,-4) \rightarrow \\
\mathcal{H}^{1}(2,-2) \oplus \mathcal{H}^{1}(2,-3) \oplus \mathcal{H}^{1}(1,-2) \rightarrow 0 .
\end{aligned}
$$


Using Theorem 4.1 we identify the only map in this complex

$$
\begin{array}{r}
\phi: S_{1}(1) \otimes S_{2}^{*}(2) \oplus S_{1}(0) \otimes S_{2}^{*}(1) \oplus S_{1}(0) \otimes S_{2}^{*}(2) \rightarrow \\
S_{1}(2) \otimes S_{2}^{*}(0) \oplus S_{1}(2) \otimes S_{2}^{*}(1) \oplus S_{1}(1) \otimes S_{2}^{*}(0)
\end{array}
$$

and we obtain the following (transposed) $3 \times 3$ block matrix containing six primal-dual multiplication blocks

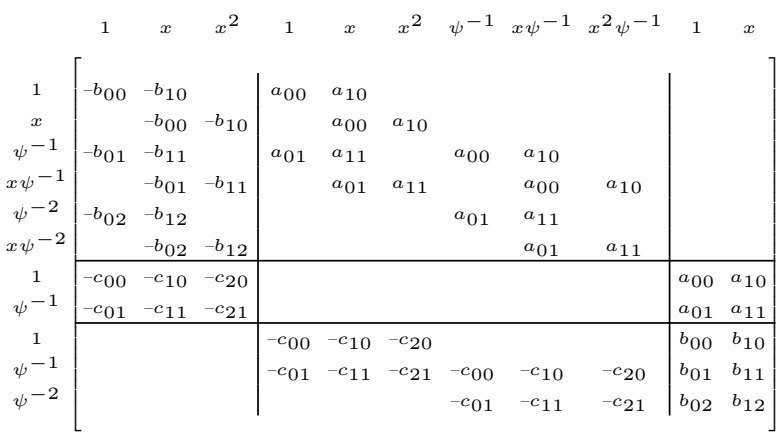

where all the missing entries are equal to zero.

In the general bivariate case the Koszul resultant matrix has a $3 \times 3$ block structure and each block $\delta_{i j}^{r}$ has dimensions $d_{r 2}\left(d_{01}+d_{11}+d_{21}-d_{r 1}\right) \times\left(d_{i 2}+d_{j 2}\right)\left(d_{01}+d_{11}+d_{21}-d_{i 1}-d_{j 1}\right)$, for $i, j \in\{0,1,2\}, i<j$ and $r \in\{i, j\}$.

\section{SOME APPLICATIONS}

In this section we use our construction for computing discriminants and mixed discriminants, as well as for computing the roots of the polynomial system (1).

\subsection{Discriminants of TP polynomials}

We present a formula for the discriminant of a bivariate tensor-product (TP) polynomial involving the Koszul resultant matrix. We consider a single TP polynomial

$$
f(x, y)=\sum_{i=0}^{d_{1}} \sum_{j=0}^{d_{2}} a_{i, j} x^{i} y^{j}
$$

of bidegree $\left(d_{1}, d_{2}\right)$. The $\left(d_{1}, d_{2}\right)$-discriminant $\Delta_{d_{1}, d_{2}}(f)$ is defined as the irreducible polynomial in $\mathbb{Z}[F]$, where $F$ stands for the coefficients of $f$, with the property that it vanishes whenever $f(x, y)$ has a singularity, that is, whenever there is a root of $f$ which satisfies

$$
f=\partial_{x} f=\partial_{y} f=0,
$$

see also [32, Chapter 12]. In the following lemma we use the notion of principal $\left(d_{1}, d_{2}\right)$-determinant [32, Chapter 12], denoted $\mathcal{E}(f)$, to relate the discriminant to our resultant construction.

Lemma 5.1. Consider the polynomial in (15) with symbolic coefficients $a_{i, j}$. The discriminant of $f(x, y)$ has degree

$$
\operatorname{deg}_{F} \Delta_{d_{1}, d_{2}}(f)=6 d_{1} d_{2}-4\left(d_{1}+d_{2}-1\right)
$$

with respect to the coefficients of $f$, and is equal to

$$
\Delta_{d_{1}, d_{2}}(f)=\frac{\mathcal{E}(f)}{a_{d_{1}, d_{2}} \Delta_{d_{2}}\left(\partial_{x}^{d_{1}} f\right) \Delta_{d_{1}}\left(\partial_{y}^{d_{2}} f\right)},
$$

where $\mathcal{E}(f)= \pm \mathcal{R}\left(f, \partial_{x} f, \partial_{y} f\right)$ is given as the determinant of the Koszul resultant matrix of Theorem 4.1.
Proof. The result follows from [32, Chapter 10,Th. 1.2], which we specialize for $f(x, y)$ in (15). That theorem states that the discriminant $\Delta_{d_{1}, d_{2}}(f)$ is related to $\mathcal{E}(f)$ via

$$
\mathcal{E}(f)= \pm a_{d_{1}, d_{2}} \Delta_{d_{2}}\left(\partial_{x}^{d_{1}} f\right) \Delta_{d_{1}}\left(\partial_{y}^{d_{2}} f\right) \Delta_{d_{1}, d_{2}}(f) .
$$

The univariate polynomials $\partial_{x}^{d_{1}} f$ and $\partial_{y}^{d_{2}} f$ correspond (geometrically) to faces of the Newton polytope (cf. [32, Chapter 10]), which, in this case, is the square $\left(d_{1}, d_{2}\right)$. Moreover, $a_{d_{1}, d_{2}}$ is the extreme vertex of the Newton polytope.

Theorem 4.1 implies that the algebraic variety $f=\partial_{x} f=$ $\partial_{y} f=0$ is characterized by the determinant of the Koszul resultant matrix for the degrees

$$
\begin{aligned}
& \boldsymbol{d}_{0}=\left(d_{1}, d_{2}\right), \\
& \boldsymbol{d}_{1}=\operatorname{deg} \partial_{x} f=\left(d_{1}-1, d_{2}\right) \text { and } \\
& \boldsymbol{d}_{2}=\operatorname{deg} \partial_{y} f=\left(d_{1}, d_{2}-1\right) .
\end{aligned}
$$

The degree of $\Delta_{d_{1}, d_{2}}(f)$ follows directly by looking at the degree of the denominator and numerator of (16).

The interest in $\mathcal{E}(f)$ stems from the fact that it possesses a determinantal representation, which is much more simple to describe and manipulate, compared to $\Delta_{d_{1}, d_{2}}(f)$ itself. Indeed, we are in position to compute $\mathcal{E}(f)$ using the Koszul resultant matrix, by plugging in the coefficients of $f$ and its partial derivatives. Lemma 5.1 allows us to compute the discriminant as a quotient of determinants. Indeed, the univariate discriminants in the denominator of (16) are computed using the Sylvester resultants $R_{y}\left(\partial_{x}^{d_{1}} f, \partial_{y} \partial_{x}^{d_{1}} f\right)$ and $R_{x}\left(\partial_{y}^{d_{2}} f, \partial_{x} \partial_{y}^{d_{2}} f\right)$, after scaling them by proper constants.

Next, we compute the discriminant of some low-degree cases, to demonstrate the use of our formula.

- Bilinear case $\left(d_{1}, d_{2}\right)=(1,1)$. We consider a bilinear polynomial, and its partial derivatives $\partial_{x} f=a_{1,0}+a_{1,1} y, \partial_{y} f=$ $a_{0,1}+a_{1,1} x$. It is well known that the discriminant of $f$ is equal to the determinant of the associated coefficient matrix of the bilinear form $f$, ie. $\Delta_{1,1}(f)=a_{0,0} a_{1,1}-a_{0,1} a_{1,0}$. We can compute the $(2 \times 2$ block $)$ matrix of

$$
\mathcal{H}^{1}(-3,0) \oplus \mathcal{H}^{1}(-2,0) \rightarrow \mathcal{H}^{1}(-2,0) \oplus \mathcal{H}^{1}(-2,1)
$$

so that

$$
\mathcal{E}(f)=\operatorname{det}\left[\begin{array}{ccc}
-a_{1,1} & a_{1,0} & a_{1,1} \\
-a_{0,1} & a_{0,0} & a_{0,1} \\
0 & a_{1,0} & a_{1,1}
\end{array}\right]=-a_{1,1} \Delta_{1,1}(f) .
$$

- Linear-quadratic case: $\left(d_{1}, d_{2}\right)=(1,2)$. We have

$$
\mathcal{E}(f)=-a_{1,2}\left(a_{1,1}^{2}-4 a_{1,2} a_{1,0}\right) \Delta_{1,2}(f)
$$

and the final discriminant polynomial is

$$
\begin{gathered}
\Delta_{1,2}(f)=a_{0,2}^{2} a_{1,0}^{2}+a_{0,0}^{2} a_{1,2}^{2}+a_{0,0} a_{1,1}^{2} a_{0,2}+a_{1,2} a_{0,1}^{2} a_{1,0} \\
-2 a_{1,0} a_{1,2} a_{0,2} a_{0,0}-a_{0,2} a_{1,0} a_{1,1} a_{0,1}-a_{0,1} a_{0,0} a_{1,1} a_{1,2} .
\end{gathered}
$$

- Biquadratic case $\left(d_{1}, d_{2}\right)=(2,2)$. Using Sylvester's matrix we compute $\Delta_{2}\left(a_{0,2}+a_{1,2} y+a_{2,2} y^{2}\right)$ and $\Delta_{2}\left(a_{2,0}+a_{2,1} x+\right.$ $\left.a_{2,2} x^{2}\right)$. The final principal discriminant is

$$
\mathcal{E}(f)=a_{2,2}\left(a_{1,2}^{2}-4 a_{2,2} a_{0,2}\right)\left(a_{2,1}^{2}-4 a_{2,2} a_{2,0}\right) \Delta_{2,2}(f)
$$

and the polynomial $\Delta_{2,2}(f)$ has 1010 non-zero coefficients.

We can generalize this approach to the computation of mixed discriminants [25, 31]. The mixed discriminant, denoted $\mathscr{D}\left(f_{1}, f_{2}\right)$, of a bivariate tensor-product system of equations $f_{1}=f_{2}=0$ is defined (under genericity assumptions) 
as the unique (up to sign) integer, irreducible polynomial in the coefficients of $f_{1}, f_{2}$ which vanishes whenever the system has a non-degenerate multiple root.

We consider the Jacobian polynomial

$$
J(x, y)=\partial_{x} f_{1} \partial_{y} f_{2}-\partial_{y} f_{1} \partial_{x} f_{2} .
$$

Clearly

$$
\operatorname{deg} J=\left(d_{11}+d_{21}-1, d_{12}+d_{22}-1\right),
$$

and $\mathscr{D}\left(f_{1}, f_{2}\right)$ is a factor of $\mathcal{R}\left(J, f_{1}, f_{2}\right)$.

As an example we consider

$$
\begin{aligned}
f_{1} & =a_{0,0}+a_{1,0} x+a_{0,1} y+a_{1,1} x y \\
f_{2} & =b_{0,0}+b_{1,0} x+b_{0,1} y+b_{1,1} x y \\
J & =a_{1,0} b_{0,1}-b_{1,0} a_{0,1}+ \\
& +\left(a_{1,0} b_{1,1}-b_{1,0} a_{1,1}\right) x+\left(a_{1,1} b_{0,1}-b_{1,1} a_{0,1}\right) y .
\end{aligned}
$$

Note that the Jacobian is missing the monomial $x y$. Nevertheless our Koszul matrix is generically non-singular. We obtain its (factorized) determinant

$\mathcal{R}\left(J, f_{1}, f_{2}\right)=-\left(a_{1,0} b_{1,1}-b_{1,0} a_{1,1}\right)\left(a_{1,1} b_{0,1}-b_{1,1} a_{0,1}\right) \mathscr{D}\left(f_{1}, f_{2}\right)$

where

$$
\begin{aligned}
\mathscr{D}\left(f_{1}, f_{2}\right) & =b_{1,0}^{2} a_{0,1}^{2}+4 a_{0,1} a_{1,0} b_{0,0} b_{1,1}+4 a_{0,0} a_{1,1} b_{0,1} b_{1,0} \\
& -2 a_{0,1} a_{0,0} b_{1,0} b_{1,1}-2 a_{0,1} b_{0,0} b_{1,0} a_{1,1}+b_{0,1}^{2} a_{1,0}^{2} \\
& -2 a_{1,0} a_{0,0} b_{0,1} b_{1,1}-2 a_{1,0} b_{0,0} b_{0,1} a_{1,1}+a_{0,0}^{2} b_{1,1}^{2} \\
& -2 b_{0,0} a_{0,0} a_{1,1} b_{1,1}-2 b_{0,1} b_{1,0} a_{1,0} a_{0,1}+b_{0,0}^{2} a_{1,1}^{2} .
\end{aligned}
$$

In [14, Example 2.3] this discriminant is computed as the hyperdeterminant of the Cayley matrix. Indeed, in their Theorem 2.1 the authors associate the mixed discriminant with the Cayley matrix, depicted in [32, Ch. 14, Prop. 1.7].

In practice, we would not expand the matrix determinant to obtain the discriminant polynomial. A rank test on the matrix suffices to decide whether the system is singular.

\subsection{Solving bivariate polynomial systems}

We can use the Koszul resultant matrix to solve a bivariate polynomial system. We sketch two approaches. Initially in both approaches we add to the system of two tensor-product bivariate polynomials, in Eq.(1), a bilinear polynomial as a $u$-polynomial (see also Example 4.2) to exploit the construction of our optimal resultant matrix. We refer the reader to [30] for a similar technique for bilinear systems.

For the first approach, we need to find a separating bilinear form, say $f_{0}$, for the system. Using the Koszul resultant matrix we can construct a rational univariate representation (RUR) of the roots of the system. For separating linear forms and RURs of bivariate systems we refer the reader to [4-6, 8, 43]. For the general construction of the RUR we refer to $[1,2,11,43]$ and for an improved version of RUR in the bivariate case to [7]. We compute RUR by modifying accordingly the algorithm in [30]. The basic block of this construction is the computation of the determinant of the resultant matrix. If the polynomials have integer coefficients with bitsize bounded by $\tau$, then we can compute the determinant with bit complexity bounded by $\widetilde{\mathcal{O}}_{B}\left(N^{3} \tau\right)$, where $N$ is the size of the resultant matrix. This leads to an algorithm that is worse by a factor of $d$ with respect to the state-of-the-art.

A second technique, perhaps more interesting, for solving consists in using eigenvalues and eigenvectors computations.
We choose one coefficient of $f_{0}$, say $a_{0,0}$, and let $C$ be the list of corresponding monomials in $x$ and $\psi$ that serve as indices for rows and columns, respectively. We reorder the resultant matrix (14) to be

$$
\left[\begin{array}{ll}
M_{1,1} & M_{1,2} \\
M_{2,1} & M_{2,2}
\end{array}\right]
$$

such that the rows, respectively the columns, of $M_{2,2}$ are indexed by the monomials in $C$. The resultant matrix has full rank and the Schur complement

$$
M_{2,2}-M_{2,1} M_{1,1}^{-1} M_{1,2}
$$

is the matrix for the multiplication by $f_{0}$. The size of $M_{2,2}$ is the number of solutions of the system, see (2). From the eigenvalues and the eigenvectors of this matrix we can recover the coordinates of the roots of the system. For instance in Example 4.2 we can collect the submatrix corresponding to the coefficient $a_{0,0}$ to obtain the list of monomials

$$
C=\left(1, x, \psi^{-1}, x \psi^{-1} ; 1\right)
$$

and we may rearrange the rows and the columns of the Koszul resultant matrix accordingly. The dimension of the block $M_{2,2}$ is $5 \times 5$ in this case, and its eigenvalues correspond to the 5 complex solutions of the system.

One significant consequence of the existence of our resultant matrix is a complexity reduction in algorithms for solving parametric polynomial systems. In the case of parameters one prefers to perform computations with matrices having entries of degree one, because otherwise the degree of the polynomials that contain the parameters increases. From this point of view, Koszul resultant matrices are the best representation one can hope for. We leave the complexity of this approach for parametric bivariate tensor-product polynomial systems as future work.

\section{Acknowledgments}

Elias Tsigaridas is partially supported by HPAC (ANR ANR11-BS02-013) and an FP7 Marie Curie Career Integration Grant. The help of the anonymous referees is gratefully acknowledged.

\section{References}

[1] M. E. Alonso, E. Becker, M.-F. Roy, and T. Wörmann. Algorithms in algebraic geometry and applications. chapter Zeros, Multiplicities, and Idempotents for Zero-dimensional Systems, pp. 1-15. 1996.

[2] A. Bostan, B. Salvy, and É. Schost. Fast algorithms for zero-dimensional polynomial systems using duality. Applicable Algebra in Engineering, Communication and Computing, 14 (4): 239-272, 2003.

[3] R. Bott. Homogeneous vector bundles. Annals of Mathematics, 66 (2): 203-248, 1957.

[4] Y. Bouzidi, S. Lazard, M. Pouget, and F. Rouillier. Rational univariate representations of bivariate systems and applications. In Proc. ACM ISSAC pp. 109-116, 2013.

[5] Y. Bouzidi, S. Lazard, M. Pouget, and F. Rouillier. Separating linear forms for bivariate systems. In Proc. ACM ISSAC pp. 117-124, New York, NY, USA, 2013.

[6] Y. Bouzidi, S. Lazard, G. Moroz, M. Pouget, and F. Rouillier. Improved algorithm for computing separating linear forms for bivariate systems. In Proc. ACM ISSAC pp. 75-82, 2014. 
[7] Y. Bouzidi, S. Lazard, G. Moroz, M. Pouget, F. Rouillier, and M. Sagraloff. Improved algorithms for solving bivariate systems via Rational Univariate Representations. Tech. report, Inria, June 2015.

[8] Y. Bouzidi, S. Lazard, M. Pouget, and F. Rouillier. Separating linear forms and rational univariate representations of bivariate systems. Journal of Symbolic Computation, 68, Part 1: 84 - 119, 2015.

[9] L. Busé. Resultants of determinantal varieties. Journal of Pure and Applied Algebra, 193 (1-3): 71 - 97, 2004.

[10] L. Busé, M. Elkadi, and B. Mourrain. Using projection operators in computer aided geometric design. Comtemporary Mathematics, 334: 321-342, 2003.

[11] J. Canny. Some algebraic and geometric computations in PSPACE. In Proc. ACM STOC, pp. 460-467, 1988.

[12] J. F. Canny, E. Kaltofen, and L. Yagati. Solving systems of nonlinear polynomial equations faster. In Proc. $A C M$ ISSAC, pp. 121-128, 1989.

[13] E. Cattani and A. Dickenstein. Solving Polynomial Equations: Foundations, Algorithms, and Applications, chapter Introduction to residues and resultants, pp. 1-61. Springer Berlin Heidelberg, Berlin, Heidelberg, 2005.

[14] E. Cattani, M. A. Cueto, A. Dickenstein, S. Di Rocco, and B. Sturmfels. Mixed discriminants. Mathematische Zeitschrift, 274 (3): 761-778, 2013.

[15] A. Chtcherba and D. Kapur. Resultants for unmixed bivariate polynomial systems produced using the Dixon formulation. Journal of Symbolic Computation, 38 (2): $915-958,2004$.

[16] A. D. Chtcherba and D. Kapur. Conditions for exact resultants using the dixon formulation. In Proc. ACM ISSAC, pp. 62-70, 2000.

[17] D. Cox and E. Materov. Tate resolutions for Segre embeddings. Algebra $\&$ Number Theory, 2 (5): 523-550, 2008.

[18] C. D'Andrea. Macaulay style formulas for sparse resultants. Transactions of the American Mathematical Society, 354 (7): 2595-2629, 2002.

[19] C. D'Andrea and A. Dickenstein. Explicit formulas for the multivariate resultant. J. Pure and Applied Algebra, 164 (1-2): 59-86, 2001.

[20] C. D'Andrea and I. Z. Emiris. Hybrid sparse resultant matrices for bivariate systems. In Proc. ACM ISSAC, pp. 24-31, 2001.

[21] A. Dickenstein and I. Z. Emiris. Multihomogeneous resultant formulae by means of complexes. Journal of Symbolic Computation, 36 (3): 317-342, 2003.

[22] A. Dickenstein, I. Z. Emiris, and A. Karasoulou. SAGA - Advances in ShApes, Geometry, and Algebra, chapter Plane Mixed Discriminants and Toric Jacobians, pp. 105-121. Springer Int'l Publishing, 2014.

[23] M. Elkadi and A. Galligo. Systems of three polynomials with two separated variables. In Proc. ACM ISSAC, pp. 159-166, 2007.

[24] I. Emiris and R. Vidunas. Root counts of semi-mixed systems, and an application to counting Nash equilibria. In Proc. ACM ISSAC, pp. 154-161, Kobe, Japan, 2014.

[25] I. Z. Emiris and A. Karasoulou. Future Vision and Trends on Shapes, Geometry and Algebra, chapter Sparse Discriminants and Applications, pp. 55-71. Springer, London, 2014.

[26] I. Z. Emiris and A. Mantzaflaris. Multihomogeneous resultant formulae for systems with scaled support. Journal of Symbolic Computation, 47 (7): 820-842, 2012.

[27] I. Z. Emiris and B. Mourrain. Matrices in elimination theory. Journal of Symbolic Computation, 28 (1): 3-44, 1999.
[28] I. Z. Emiris and V. Y. Pan. Symbolic and numeric methods for exploiting structure in constructing resultant matrices. Journal of Symbolic Computation, 33 (4): 393-413, Apr. 2002.

[29] I. Z. Emiris and V. Y. Pan. Improved algorithms for computing determinants and resultants. Journal of Complexity, 21 (1): 43-71, Feb. 2005.

[30] I. Z. Emiris, A. Mantzaflaris, and E. Tsigaridas. On the bit complexity of solving bilinear polynomial systems. In Proc. ACM ISSAC, pp. 215-222, 2016.

[31] A. Esterov. The discriminant of a system of equations. Advances in Mathematics, 245: 534 - 572, 2013.

[32] I. Gelfand, M. Kapranov, and A. Zelevinsky. Discriminants, Resultants and Multidimensional Determinants. Birkhäuser, Boston, 1994.

[33] R. Hartshorne. Algebraic Geometry. Springer, New York, 1977.

[34] A. Khetan. The resultant of an unmixed bivariate system. Journal of Symbolic Computation, 36 (3-4): 425442, 2003.

[35] A. Khetan, N. Song, and R. Goldman. Sylvester Aresultants for bivariate polynomials with planar Newton polygons. In Proc. ACM ISSAC, pp. 205-212, 2004.

[36] F. S. Macaulay. Some formulæ in elimination. Proceedings of the London Mathematical Society, s1-35 (1): 3-27, 1902.

[37] A. Mantzaflaris and B. Mourrain. Deflation and certified isolation of singular zeros of polynomial systems. In Proc. ACM ISSAC, pp. 249-256, 2011.

[38] A. Mantzaflaris and B. Mourrain. Singular zeros of polynomial systems. In T. Dokken and G. Muntingh, editors, Advances in Shapes, Geometry, and Algebra, volume 10 of Geometry and Computing, pp. 77-103. Springer, 2014.

[39] N. McCoy. On the resultant of a system of forms homogeneous in each of several sets of variables. Trans. $A M S, 35$ (1): 215-233, 1933.

[40] A. McLennan. The maximum number of real roots of a multihomogeneous system of polynomial equations. Contributions to Algebra and Geometry, 40 (2): 343350, 1999.

[41] E. Miller and B. Sturmfels. Combinatorial commutative algebra, volume 227. Springer, 2004.

[42] T. Muir. The resultant of a set of lineo-linear equations. Proc. Royal Soc. of South Africa, 2 (1): 373-380, 1910.

[43] F. Rouillier. Solving zero-dimensional systems through the rational univariate representation. Applicable Algebra in Engineering, Communication and Computing, 9 (5): 433-461, 1999.

[44] H. Schenck, D. Cox, and A. Dickenstein. A case study in bigraded commutative algebra. Syzygies 8 Hilbert Functions, 254: 67-112, 2007.

[45] B. Sturmfels and A. Zelevinsky. Multigraded resultants of Sylvester type. Journal of Algebra, 163 (1): 115-127, 1994.

[46] J. Sylvester. On the degree and weight of the resultant of a multipartite system of equations. Proc. Royal Soc. of London, 12: 674-676, 1862.

[47] J. Weyman. Calculating discriminants by higher direct images. Transactions of AMS, 343 (1): 367-389, 1994.

[48] J. Weyman. Cohomology of Vector Bundles and Syzygies. Cambridge University Press, 2003.

[49] J. Weyman and A. Zelevinsky. Determinantal formulas for multigraded resultants. Journal of Algebraic Geometry, 3 (4): 569-597, 1994.

[50] M. Zhang and R. Goldman. Rectangular corner cutting and Sylvester A-resultants. In Proc. ACM ISSAC, pp. 301-308, 2000. 